J. RÖDER

H.-U. KREBS

\section{Frequency dependent smoothing of rough surfaces by laser deposition of $\mathrm{ZrO}_{2}$}

Institut für Materialphysik, University of Göttingen, Friedrich-Hund-Platz 1, 37077 Göttingen, Germany
Received: 10 December 2007/Accepted: 12 December 2007

Published online: 11 January 2008 • () The Author(s) 2008

ABSTRACT The development of the surface roughness of amorphous $\mathrm{ZrO}_{2}$ layers with different thicknesses, which were laser deposited on nanocrystalline Ag surfaces, was analyzed by atomic force microscopy. With increasing $\mathrm{ZrO}_{2}$ layer thickness first the surface slightly roughens due to island growth, but above a layer thickness of about $30 \mathrm{~nm}$ it continuously smoothens. From the power spectral densities it is clear that the smoothing processes are frequency dependent. First the high-frequency surface features vanish before lower roughness frequencies are decreased. Although the starting roughness is only $1 \mathrm{~nm}$, a $\mathrm{ZrO}_{2}$ layer thickness of about $4 \mu \mathrm{m}$ is necessary to smooth the long-wavelength surface features. From the decrease of the roughness with $\mathrm{ZrO}_{2}$ layer thickness and the observed final spectral densities, the dominant smoothing mechanisms were identified as downhill currents, probably induced by the energetic ions impinging on the substrate surface during deposition.

PACS 68.35.Ct; 68.37.Ps

\section{Introduction}

Nowadays the investigation of growth characteristics is of great interest, as for many thin film technologies the surface morphology plays an important role. Especially for optical, electrical or mechanical properties it is important to control features like the film thickness, grain sizes, as well as surface and interface roughness. Well investigated examples are X-ray mirrors consisting of multilayer structures with very smooth interfaces [1]. Here the reflectivity as well as the optical resolution decreases with increasing interface roughness. In systems with magnetoresistive effects like giant or tunnelling magnetoresistance, an optimal control of the roughness is essential, as the scattering of electrons depends strongly on the properties of the interfaces $[2,3]$.

Up to now a lot of experimental and theoretical work has been done to in- vestigate the roughness evolution during film growth on smooth substrates, where a scale dependent behaviour of the roughness of the following form is predicted

$$
\begin{gathered}
\sigma(L=\text { const }, t) \propto \\
\left\{\begin{array}{lll}
t^{\beta} \propto d^{\beta} & \text { for } t \ll t_{c} \\
\text { const } & \text { for } t \gg t_{c}
\end{array}\right.
\end{gathered}
$$

Here $t$ denominates the deposition time, $L$ the scan size, $d$ the deposited film thickness and $\beta$ the growth exponent. In contrast, only a few publications deal with the growth of films on a rough surface, where smoothing phenomena may occur depending on the materials used. Such experiments also allow the investigation of the dominating growth mechanisms during thin film deposition.

In general a promising tool to deal with different growth processes are stochastic differential equations, where interfaces are described on a mesoscopic scale and atomic processes are neglected. A general equation is

$\frac{\partial h(\boldsymbol{x}, t)}{\partial t}=G[h(\boldsymbol{x}, t)]+\eta(\boldsymbol{x}, t)$,

where $h(\boldsymbol{x}, t)$ describes the relative height of the surface, $G[h(x, t)]$ is a functional, whose contributions arise from $h(\boldsymbol{x}, t)$ and its spacial derivations, and $\eta(\boldsymbol{x}, t)$ is white noise [4].

As in many cases a direct solution of this equation is not possible, one can transform this equation in Fourier space. This can especially be suitable if $G[h(\boldsymbol{x}, t)]$ is linear in $h$. One obtains

$$
\begin{aligned}
& \frac{\partial \hat{h}(\boldsymbol{k}, t)}{\partial t}=-\sum_{i} b_{i} k^{i} \hat{h}(\boldsymbol{k}, t)+\hat{\eta}(\boldsymbol{k}, t) \\
& i \in \mathbb{N} .
\end{aligned}
$$

Here $\boldsymbol{k}$ is the wave vector, $b_{i}$ are constants indicating the significance of the related terms, and $\hat{h}(\boldsymbol{x}, t), \hat{\eta}(\boldsymbol{x}, t)$ denotes the Fourier transformation of the respective functions. The first four terms of the polynomial can be related to different transport mechanisms like viscous flow $(i=1)$, desorption $(i=2)$, volume diffusion $(i=3)$ and surface diffusion $(i=4)[5,6]$. The term for $i=2$ can furthermore be identified with downhill currents due to ion impacts [7], shadowing effects [8] or sputter erosion [9]. If the respective effects contribute to smoothing, $b$ is positive. Otherwise in the case of roughening phenomena it is negative. In the deterministic case (that means without white noise) and for an initially flat surface this equation can be solved by the ansatz

$\hat{h}(\boldsymbol{k}, t)=\hat{h}(\boldsymbol{k}, t=0) \exp \left(-t \sum_{i} b_{i} k^{i}\right)$, 
and the power spectral density can be calculated by the function:

$\operatorname{PSD}(k, t)=R(k) \frac{1-\exp \left(-2 t \sum b_{i} k^{i}\right)}{\sum b_{i} k^{i}}$,

where $R(k)$ is the strength of the noise [10]. In case of white noise, which is uncorrelated in space and time, $R$ is a constant.

Furthermore, the roughness is obtained from the power spectral density by the following equation:

$\sigma_{\mathrm{rms}}^{2}(t)=2 \pi \int_{0}^{\infty} k \cdot \operatorname{PSD}(k, t) \mathrm{d} k$.

In the case that roughening during growth is only caused by uncorrelated noise, $\operatorname{PSD}(k, t)$ is independent of $k$. Then (6) can be simplified for each smoothing process of the order $i$ to [11]

$\sigma_{\mathrm{rms}}(t) \propto t^{-1 / i}$.

When studying the smoothing mechanisms during film deposition it is helpful to start with a statistically rough surface, where all kind of spacial frequencies are present. Then it is obvious from (3) that smoothing depends on frequency and hence should start at high frequencies.

One possibility to get a surface with sufficiently high roughness is to deposit a material like $\mathrm{Ag}$ on a $\mathrm{Si}$ substrate. Ag shows island growth with large grains due to high diffusivity. As smoothing material an oxide should be suitable, as this material class is characterized by a much lower surface energy than metals have, and often shows a smooth surface. In our case $\mathrm{ZrO}_{2}$ is chosen as the smoothing material, because it has been shown that nearly totally smooth films can be grown on Si [12].

The aim of this paper is to study the successive smoothing of a rough $\mathrm{Ag}$-surface by depositing an increasing amount of $\mathrm{ZrO}_{2}$. First the properties of the rough Ag-films on $\mathrm{Si}$ are presented. Then, the successive smoothing of the rough $44 \mathrm{~nm}$ thick Ag-films by $\mathrm{ZrO}_{2}$ is described, qualitatively as well as quantitatively. It will be shown that the smoothing indeed is frequency dependent, and the main smoothing mechanisms will be identified.

\section{Experimental setup}

All samples were prepared by pulsed laser deposition (PLD) at room temperature in an ultra high vacuum chamber with a base pressure of $10^{-8}$ mbar. For ablation a $\mathrm{KrF}$ excimer laser was used with a wavelength of $248 \mathrm{~nm}$, pulse duration of $30 \mathrm{~ns}$ and repetition rate of $10 \mathrm{~Hz}$. The $\mathrm{ZrO}_{2}$ target consisted of $\mathrm{ZrO}_{2}$ with $3 \mathrm{~mol} . \%$ $\mathrm{Y}_{2} \mathrm{O}_{3}$. Both $\mathrm{Ag}$ and $\mathrm{ZrO}_{2}$ were ablated at a laser fluence of $6 \mathrm{~J} / \mathrm{cm}^{2}$ on $\mathrm{Si}(111)$ substrates at a target-to-substrate distance of $50 \mathrm{~mm}$. The average deposition rate was determined by profilometry. The total thicknesses of the rough Agfilm or the smoothing $\mathrm{ZrO}_{2}$ films on top are given by the number of laser pulses. The setup has been already described in detail earlier [13].

The surfaces of the samples were investigated by atomic force microscopy (AFM) using a Nanoscope 4 (Veeco) operating in tapping mode. A Si tip was used with an average radius of about $10 \mathrm{~nm}$, the scanned area ranged from $0.5 \times 0.5 \mu \mathrm{m}^{2}$ to $5 \times 5 \mu \mathrm{m}^{2}$ with a resolution of $512 \times 512$ data points in each case. The rms-roughness of the film surfaces and the corresponding power spectral densities (PSD) were calculated and compared for $1 \times 1 \mu \mathrm{m}^{2}$ images using the analysis softwares V530 and V613 of Veeco. The slopes of the power spectral densities are investigated as well as the bends appearing in the spectra, which can be identified as lateral correlation lengths.

\section{$3 \quad$ Results and discussion}

First, the roughness of the Ag-film is characterized to define the initial conditions for smoothing. To

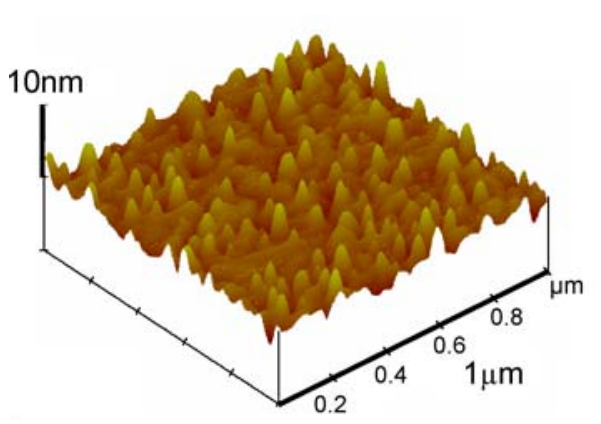

FIGURE 1 (a) AFM picture of a $44 \mathrm{~nm}$ thick Ag film on $\mathrm{Si}(111)$; (b) power spectral densities of different Ag layers of the same thickness study the growth of $\mathrm{Ag}$ on $\mathrm{Si}$, several films of different thicknesses were laser deposited and analyzed via AFM. It was found (details will be published elsewhere) that Ag shows distinct formation of isolated islands, growth of these islands and then coalescence phenomena up to an average layer thickness of a few nanometers. Above a thickness of about $8 \mathrm{~nm}$, the films are closed, a crossover from island to columnar growth takes place and the film roughness increases with increasing layer thickness.

At a layer thickness of $44 \mathrm{~nm}$, a rmsroughness of about $0.8 \pm 0.1 \mathrm{~nm}$ is obtained which seemed appropriate for the smoothing experiments discussed below. In Fig. 1a an AFM image of such a $44 \mathrm{~nm}$ thick Ag-film deposited on $\mathrm{Si}$ is shown. The spatial distribution of the roughness as well as the correlations between the roughness and specific surface features were investigated calculating the power spectral densities of the AFM images. In Fig. 1b the PSDs of four different films of equal thickness can be compared. It is important to note that for $1 \times 1 \mu \mathrm{m}^{2}$ scans the PSDs are not very reproducible for spacial frequencies of less than $10^{-2} \mathrm{~nm}^{-1}$ (corresponding to a spatial wavelength of $0.1 \mu \mathrm{m})$, because not enough data points are available in the averaging procedure to obtain adequate statistics. Thus a lower interpretation-limit for the PSDs is given. An upper limit exists due to the finite tip radius. In our case this is the frequency of $1 / R_{\text {tip }}=10^{-1} \mathrm{~nm}^{-1}$.

The PSD graphs show a bend at low frequencies, where the initial region with horizontal slope of the spectra is followed by a scaling range over which the PSD decreases with a slope of $y=-5.7$. The transition occurs at

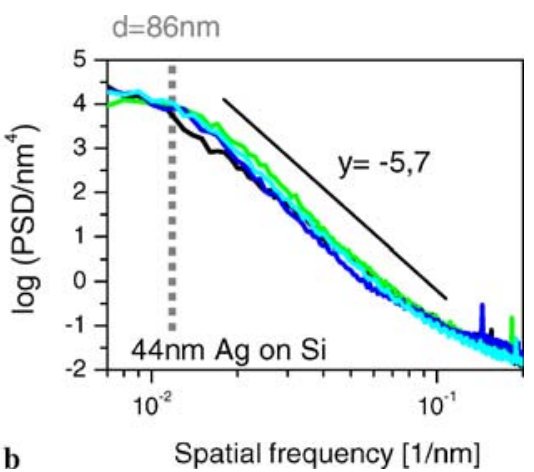


a cut-off frequency $k_{\mathrm{c}}$ (dotted line), corresponding to a scaling length $\xi=$ $1 / k_{\mathrm{c}}$ of $86 \pm 5 \mathrm{~nm}$, which is called correlation length and can be identified with the crystal grain size seen in the AFM images [14,15]. The observed fast decay of the spectra with a slope of -5.7 is quite uncommon. In the case of amorphous surfaces, where diffusion, condensation or shadowing are the dominant growth processes, normally a minimum value of -4 is obtained. But, higher exponents have already been seen for instance by Eisenmenger-Sittner, who similar to our experiments showed for the deposition of crystalline $\mathrm{Al}$ on amorphous substrates that a decrease in the range of -5 can be attributed to three-dimensional island growth and coalescence phenomena [16]. Also in our case, where the Ag layers are closed, sharp edges occurring in the columnar structures between the columns and grain boundaries are probably responsible for the high slope in the spectra, which can not be interpreted any further by using the classic roughening models described before.

The growth of $\mathrm{ZrO}_{2}$ on smooth $\mathrm{Si}(111)$-substrates was studied to obtain information about the roughness formation of this material. Pulsed laser deposition of $\mathrm{ZrO}_{2}$ films has already been investigated in the literature [17] leading at room temperature to very smooth amorphous films [18]. Also in our case amorphous growth is observed and all investigated films showed the same small rms-roughness of about $0.1 \pm 0.03 \mathrm{~nm}$, independently of the layer thickness. Scaling laws during growth could not be observed. It is interesting to note that even the $\mathrm{Si}$-substrates with the low rms-roughness of $0.3 \mathrm{~nm}$ are smoothed by the deposition of $\mathrm{ZrO}_{2}$ layers.

In the following, smoothing of the rough $\mathrm{Ag}$ surface by deposition of $\mathrm{ZrO}_{2}$ was studied. Therefore, $\mathrm{ZrO}_{2}$ films of different thicknesses between $2 \mathrm{~nm}$ and $960 \mathrm{~nm}$ were deposited on $44 \mathrm{~nm}$ thick Ag-films. In Fig. 2 four AFM images are presented showing the surfaces after smoothing with $2 \mathrm{~nm}, 45 \mathrm{~nm}, 170 \mathrm{~nm}$ and $370 \mathrm{~nm}$ of $\mathrm{ZrO}_{2}$, respectively. It can directly be seen in the AFM images that the heights of the hills decrease with increasing $\mathrm{ZrO}_{2}$ amount indicating a successive smoothing of the surface. It should also be noticed that simultan-
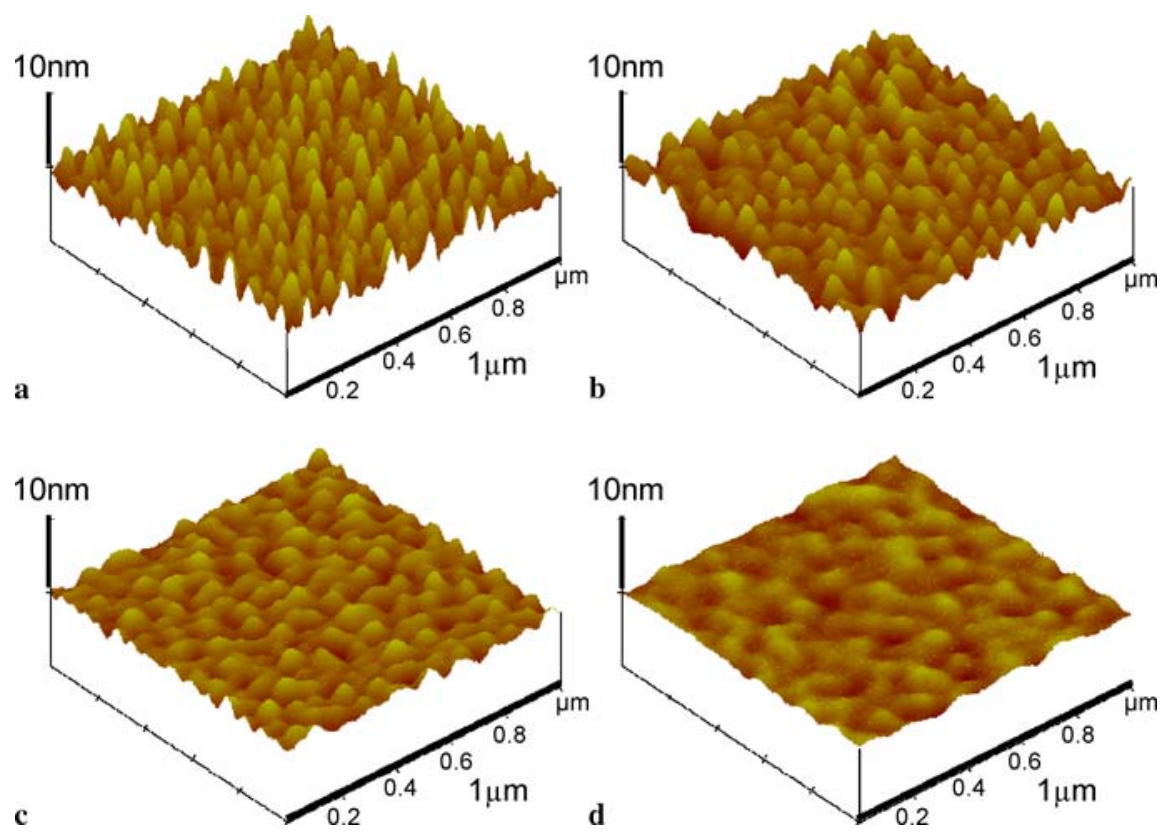

FIGURE 2 AFM images of $44 \mathrm{~nm}$ thick $\mathrm{Ag}$ films after deposition of $\mathrm{ZrO}_{2}$ with a layer thickness of (a) $1.7 \mathrm{~nm}$, (b) $45 \mathrm{~nm}$, (c) $170 \mathrm{~nm}$ and (d) $370 \mathrm{~nm}$

eously with the decrease of the roughness the lateral width of the islands increases.

For a better overview some crosssection lines of different samples are summarized in Fig. 3. Here the same trends are visible, the roughness decreases with increasing $\mathrm{ZrO}_{2}$ thickness. However, it is interesting to note that the hills of the pure Ag-film are slightly smaller than those with a small $\mathrm{ZrO}_{2}$ layer on top indicating that first a local roughening effect occurs before the surface is globally smoothed at larger film thicknesses.

The development of the rms-roughness vs. $\mathrm{ZrO}_{2}$ film thickness is shown in Fig. 4a in more detail. It can clearly be seen that first the roughness increases

from $0.8 \mathrm{~nm}$ of the pure $\mathrm{Ag}$ film to about $1.1 \mathrm{~nm}$ for low $\mathrm{ZrO}_{2}$ layer thicknesses. This could be explained assuming that in the early growth stages probably amorphous $\mathrm{ZrO}_{2}$ islands are formed on top of the crystalline Ag grains increasing the surface roughness. Above $\mathrm{ZrO}_{2}$ layer thickness of $30 \mathrm{~nm}$ the roughness continuously decreases with a slope of -0.5 . This decay indicates that a mechanisms of the order of $i=2$ is dominant during surface smoothing (see (7)).

To investigate the changes of the roughness in dependence on the evolution of the lateral structures on the surface, again the power spectra of the images were calculated and for better comparison plotted in one graph (see Fig. 5). It can be seen that in the main the

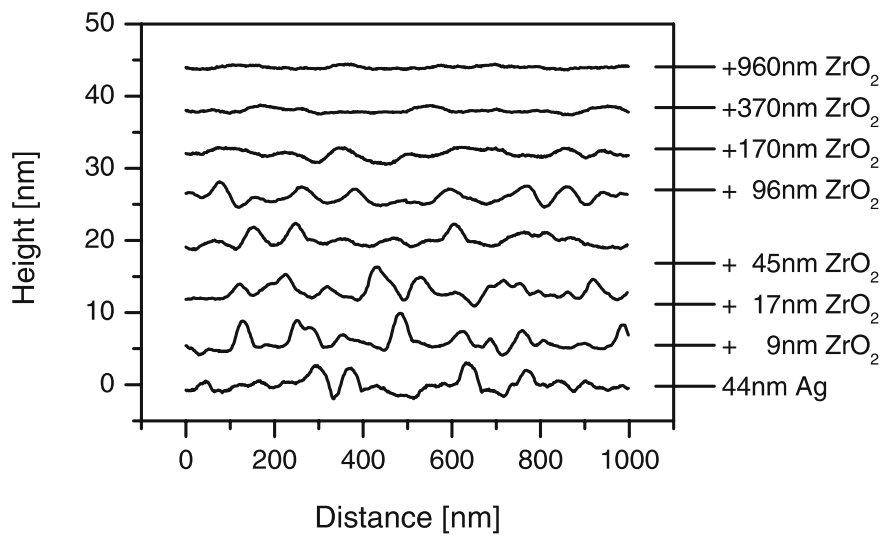

FIGURE 3 Cross-section-plots of different samples, each smoothed with the noted layer thickness of $\mathrm{ZrO}_{2}$ 

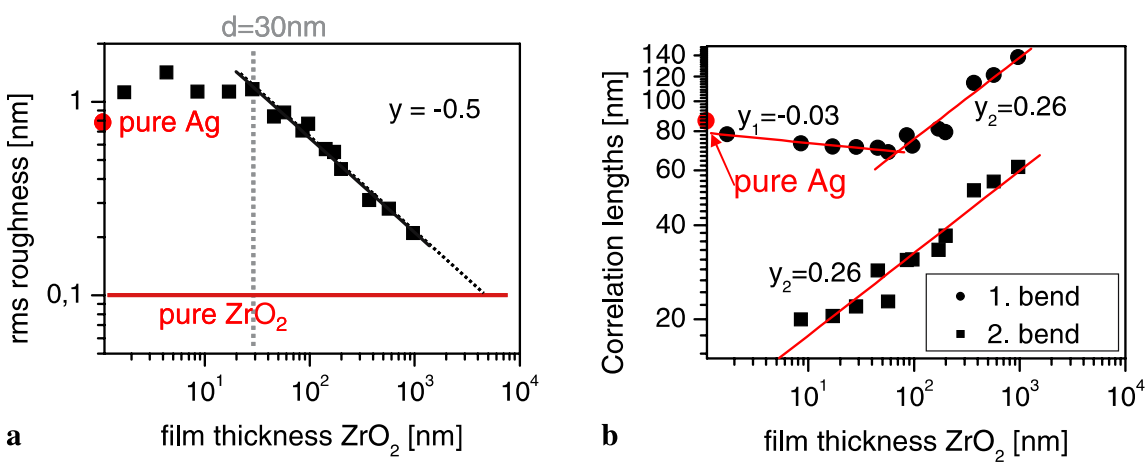

FIGURE 4 (a) RMS-roughness of the smoothed samples vs. film thickness of the $\mathrm{ZrO}_{2}$-layer; (b) correlation lengths calculated from the two bends in the PSDs vs. film thickness of the $\mathrm{ZrO}_{2}$-layer

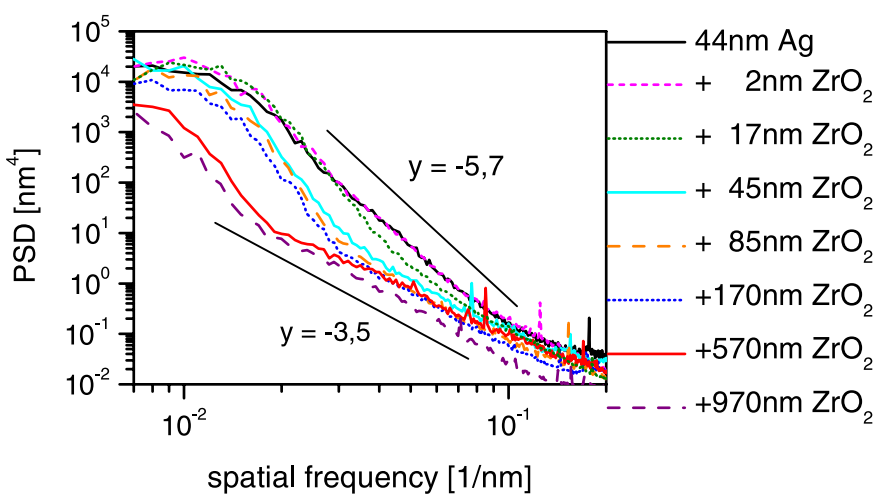

FIGURE 5 Power spectral densities of the original Ag film and successively smoothed films covered by $\mathrm{ZrO}_{2}$. For the last film a fit of the high frequency part of the spectrum is shown

spectra decrease with higher amounts of $\mathrm{ZrO}_{2}$. This is understandable, as in the case of an isotropic surface the area under the PSD curve is connected with the surface roughness $\sigma$ according to (6). Thus the decrease of the curves is due to successive smoothing of the initial rough $\mathrm{Ag}$ surface. In the PSD spectra, the initial increase of the surface roughness for thin $\mathrm{ZrO}_{2}$-layers is connected with a slight increase in the low frequency range again indicating that in the initial stages island growth of $\mathrm{ZrO}_{2}$ on Ag takes place. Then, with increasing amounts of $\mathrm{ZrO}_{2}$, the high frequency part of the PSD spectra is reduced successively, while lower spatial frequencies are less decimated. This clearly indicates that smoothing indeed occurs as frequency dependent as expected from (3). Higher frequencies are smoothed out quite fast, whereas lower frequencies need larger $\mathrm{ZrO}_{2}$ layer thicknesses for smoothing.

For a more detailed analysis the two bends of the PSD spectra are considered. The first one between the initial region with a horizontal slope and the region, where a scaling behaviour is observed, has already been discussed above for pure Ag films, and can again be interpreted as the dominating, characteristic correlation length on the surface. It can be attributed to the lateral diameter of the amorphous hills. The second bend determines the spatial frequency, up to that smoothing has taken place for higher frequencies. In Fig. 4b the position of both bends are plotted vs. the $\mathrm{ZrO}_{2}$-film thickness. It can be seen, that the correlation length calculated from the position of the first bend keeps more or less the same for small thicknesses and then crosses over to a scaling range regime increasing with a slope of 0.26 . The second bend only occurs for films which were smoothed with more than $5 \mathrm{~nm} \mathrm{ZrO}_{2}$ and its position shifts to lower frequencies (higher correlation lengths) with increasing $\mathrm{ZrO}_{2}$ thickness. Figure $4 \mathrm{~b}$ shows that the shift to smaller frequencies exhibits the same scaling behaviour as that of the first bend, namely a slope of 0.26 .

The second bend in the spectra limits a range at high frequencies, where a scaling behaviour with a slope of -3.5 is observed. This is the same slope that pure $\mathrm{ZrO}_{2}$ on $\mathrm{Si}$ shows. As the PSD represents the spatial frequency content of the surface roughness, its very low values on the right hand side of the second bend imply that these frequencies already have been smoothed as much as possible with $\mathrm{ZrO}_{2}$ as the smoothing material. In this context we interpret the transition wavelength as the biggest structure size which already has been smoothed. As there is no strict limit in which frequency range smoothing phenomena occur, the transition to low frequencies, which were not smoothed yet, is washed out. The growth behaviour of the system becomes more and more similar to the deposition of $\mathrm{ZrO}_{2}$ on the flat $\mathrm{Si}$ surface, but it can be seen in the power spectra, that a film thickness of $960 \mathrm{~nm}$ is insufficient to smooth the system completely, as in the low frequency/high wavelength range (above $\sim 70 \mathrm{~nm}$ ) a certain amount of frequencies still contribute to the roughness. As indicated by an extrapolation of the straight line fitted to the roughness values of Fig. $4 \mathrm{a}$, a $\mathrm{ZrO}_{2}$ film thickness of about $4 \mu \mathrm{m}$ would be necessary for completely smoothing out the initial roughness of the Ag film, of only $0.8 \mathrm{~nm}$.

Also from the value of the final slope in the PSD spectra of -3.5 (for higher frequencies) the dominant smoothing mechanisms can be obtained. For this, the corresponding part of the last spectrum was fitted by using (5). As the low frequency range is not totally smoothed yet, this area was not considered. The relative importance of different mechanisms can be estimated by fitting the prefactors $b_{i}$ and calculating the quotient $b_{i} k^{i} / b_{j} k^{j}$. The results of the fit are shown in Fig. 6 in comparison to the fit of a pure $\mathrm{ZrO}_{2}$ layer on $\mathrm{Si}$, where similar relations are obtained. For all fits the mechanisms of the order $i=1$ (viscous flow) and $i=3$ (volume diffusion) could be neglected. This is not surprising as viscous flow only occurs for very high ion energies $(\mathrm{MeV})$, and volume diffusion (as a material transport mechanisms) is much lower than surface diffusion in solids. All fits show that the dominant smoothing mechanism for wavelengths higher than $40 \mathrm{~nm}$ is of the order $i=2$, because the prefactor $b_{2}$ is 2-3 orders of magnitude higher than $b_{4}$.

The smoothing mechanisms related to $i=2$ are desorption $[5,6]$, shadowing effects [8], or so called downhill 


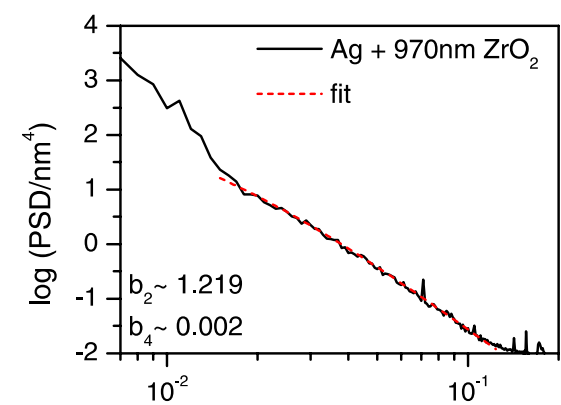

a

spatial frequency $[1 / \mathrm{nm}]$

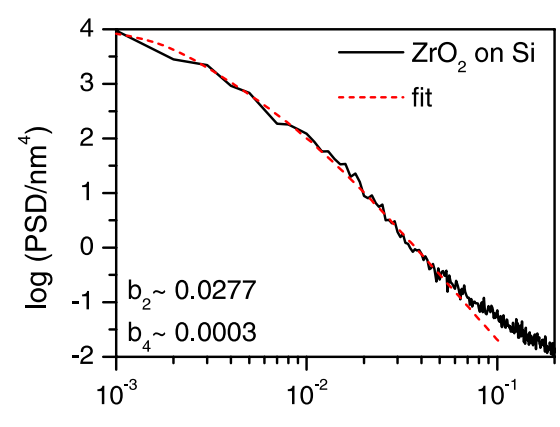

b spatial frequency $[1 / \mathrm{nm}]$

FIGURE 6 (a) Fits of the high frequency part of the spectrum of an Ag film smoothed with a $970 \mathrm{~nm}$ thick $\mathrm{ZrO}_{2}$ layer; (b) fit of a pure $\mathrm{ZrO}_{2}$ film on $\mathrm{Si}$. The prefactor $b_{2}$ is always 2-3 orders of magnitude higher than $b_{4}$

currents [7]. To decide which mechanisms is valid in our case, one has to keep in mind that during PLD ions with high energies up to some hundreds of $\mathrm{eV}$ impinge on the film [19] and are implanted a few nanometers below the surface, enhancing the mobility of atoms and inducing atom rearrangements within the surface layers (subplantation, see [20]). Desorption as a thermal process is marginal and shadowing effects seem quite unlikely under these conditions of high particle energies. However, as Moseler et al. described for amorphous films, ion energies of about $30 \mathrm{eV}$ are sufficient to produce downhill currents after the impact, which leads to a smoothing of initially rough substrates by the erosion of hills [7]. Therefore, in agreement with the decay of the roughness with a power of -0.5 (Fig. 4a) the dominant smoothing mechanisms are identified as downhill currents $(i=2)$. Nevertheless, also surface diffusion $(i=4)$ processes take place and have to be taken into account, because negative slopes higher than -2 are obtained. However they are only important for small surface features $(<40 \mathrm{~nm})$, which are smoothed out fast at low layer thicknesses. For the smoothing of bigger surface structures, surface diffusion is a subordinate mechanism as seen in the quotient of the prefactors.

\section{Conclusions}

In summary, it was shown that rough Ag surfaces can be smoothed by deposition of $\mathrm{ZrO}_{2}$ layers of different thicknesses. The surface first develops a roughening due to the growth of amorphous islands up to a layer thickness of about $30 \mathrm{~nm}$, before smoothing starts to dominate. It was clearly observed that smoothing of the surface takes place as frequency-dependent. Initially, high frequencies are smoothed, before lower frequencies are decreased with increasing $\mathrm{ZrO}_{2}$ film thickness. Altogether a layer thickness of about $4 \mu \mathrm{m}$ is necessary to completely smooth the low-frequency surface features, although the initial roughness was only about $1 \mathrm{~nm}$. From the decrease of the roughness and the final spectral densities the dominant smoothing mechanisms were identified as downhill currents probably induced by the energetic ions impinging the substrate surface during laser deposition.
ACKNOWLEDGEMENTS We thank S. Mayr and S. Vauth for fruitful discussions. This work was supported by the Deutsche Forschungsgemeinschaft (SFB 602).

OPEN ACCESS This article is distributed under the terms of the Creative Commons Attribution Noncommercial License which permits any noncommercial use, distribution, and reproduction in any medium, provided the original author(s) and source are credited.

\section{REFERENCES}

1 T. Salditt, D. Lott, T.H. Metzger, J. Peisl, G. Vignaud, P. Hoghoj, O. Schärpf, P. Hinze, R. Lauer, Phys. Rev. B 54, 5860 (1996)

2 E.E. Fullerton, D.M. Kelly, J. Gimpel, I.K. Schuller, Phys. Rev. Lett. 68, 859 (1992)

3 J. Faupel, H.-U. Krebs, A. Käufler, Y. Luo, K. Samwer, S. Vitta, J. Appl. Phys. 92, 1171 (2002)

4 A.-L. Barabási, H.E. Stanley, Fractal Concepts of Surface Growth (Cambridge University Press, Cambridge, 1995)

5 W.W. Mullins, J. Appl. Phys. 28, 333 (1957)

6 C. Herring, J. Appl. Phys. 21, 301 (1950)

7 M. Moseler, P. Gumbsch, C. Casiraghi, A.C. Ferrari, J. Robertson, Science 309, 1545 (2005)

8 N.J. Sevchik, J. Non-Cryst. Solids 12, 141 (1973)

9 R.M. Bradley, J.M.E. Harper, J. Vac. Sci. Technol. A 6, 2390 (1988)

10 J. Villain, J. Phys. I 1, 19 (1991)

11 S. Vauth, PhD Theses, University of Göttingen, Germany (2008)

12 J.P. Chang, Y.-S. Lin, K. Chu, J. Vac. Sci. Technol. B 19, 1782 (2001)

13 H.-U. Krebs, J. Non-Equil. Process. 10, 3 (1997)

14 K. Zhu, V. Kuryatkov, B. Borisov, J. Yun, G. Kipshidze, S.A. Nikishin, H. Temkin, D. Aurongzeb, M. Holtz, J. Appl. Phys. 95, 4635 (2004)

15 D. Aurongzeb, M. Holtz, L. Menon, Appl. Phys. Lett. 89, 092501 (2006)

16 C. Eisenmenger-Sittner, J. Appl. Phys. 89, 6085 (2001)

17 A.A. Voevodin, J.G. Jones, J.S. Zabinski, J. Appl. Phys. 88, 1088 (2000)

18 A.A. Voevodin, J.G. Jones, J.S. Zabinski, J. Vac. Sci. Technol. A 19, 1320 (2001)

19 S. Fähler, H.U. Krebs, Appl. Surf. Sci. 96-98, 61 (1996)

20 S. Fähler, M. Weisheit, K. Sturm, H.U. Krebs, Appl. Phys. A 69, 459 (1999) 